\title{
La mujer en Derecho a una Vida libre de violencia desde la Organización Comunitaria
}

\author{
Julio Juvenal Aldana Zavala \\ julioaldanazavala@gmail.com \\ https://orcid.org/0000-0002-7934-9103 \\ Universidad Nacional Experimental Francisco de Miranda \\ Venezuela \\ Elia Analy Valles González \\ analy15tutu@gmail.com \\ Universidad Nacional Experimental Francisco de Miranda \\ Venezuela
}

Recibido: 17 de noviembre de 2017

Aprobado: 26 de febrero de 2018

\begin{abstract}
RESUMEN
El presente artículo tipo ensayo, proyecta una alternativa para prevenir la violencia contra la mujer, no solo desde el punto de vista informativo, sino, formativo, es decir, se proponen los comité jurídicos en las comunidades, como organizaciones integrales por medio de las cuales, se pueden articular acciones que beneficien a la mujer desde el punto de vista psicológico, legal, laboral, educativo, como ejes medulares para el fomento de una visión de paz, en donde la familia como médula de la sociedad sea librada de la violencia en sus diversas formas. El comité jurídico para la defensa de la mujer en las comunidades, se constituye en una organización con el propósito de brindar la ayuda, atención, necesaria sobre todo a la mujer que ha sufrido violencia con la intención de apoyarle a superar los traumas que pueda padecer, así como incentivarla a creer en sí misma para constituirse en una persona innovadora, productiva, emprendedora, en capacidad de asumir los retos familiares, sociales, económicos, para ser independiente en pro de contribuir tanto a su familia como a la sociedad venezolana. Así mismo, el comité proyecta la educación preventiva a las mujeres para que eviten ser víctimas de la violencia, puedan ser promotoras en el progreso de la nación desde una perspectiva de sanidad integral en su ser y se integren al campo laboral en las mejores condiciones posibles de ser competitivas - exitosas. Por consiguiente, el comité jurídico es un espacio que contribuye en fortalecer las políticas públicas en concordancia con las leyes vigentes, siendo un aliado para generar una cultura de paz.
\end{abstract}

Descriptores: comité jurídico; violencia contra la mujer; cultura de paz. 


\title{
The woman in Right to a Life free of violence \\ from the Community Organization
}

\begin{abstract}
The present essay article proposes an alternative to prevent violence against women, not only from the information point of view, but also, formative, that is, the legal committees are proposed in the communities as integral organizations through which, It is possible to articulate actions that benefit the woman from the psychological, legal, labor, educational point of view, as central axes for the promotion of a vision of peace, in which the family as marrow of society is freed from violence in Its various forms. The legal committee for the defense of women in the communities, is an organization with the purpose of providing the assistance, attention, necessary especially to the woman who has suffered violence with the intention of supporting him to overcome the traumas that may suffer, As well as to encourage her to believe in herself to become an innovative, productive, enterprising person capable of assuming the family, social and economic challenges to be independent in order to contribute to both her family and Venezuelan society. Likewise, the committee projects preventive education to women so that they avoid being victims of violence, they can be promoters in the progress of the nation from a perspective of integral health in their being and integrate to the labor field in the best possible conditions To be competitive - successful. Therefore, the legal committee is a space that contributes to strengthen public policies in accordance with the laws in force, being an ally to generate a culture of peace.
\end{abstract}

Descriptors: legal committee; violence against women; peace culture.

\section{A MODO INTRODUCTORIO}

La violencia en contra de la mujer, es un acto con vertientes diversas y consecuencias, es decir, no hay exclusividad en la forma o modo en que la mujer sea violentada, aunque existan causas de mayor ocurrencia hacia un determinado tipo. Contrarrestar la violencia en contra de la mujer, implica trabajar día a día en fomentar una cultura de prevención en favor del género y de su dignidad como persona. Esta es una acción que desde el medioevo la mujer ha realizado, siendo muchas veces infructuosa a causa de la cultura patriarcal - machista dominante, lo cual ha impedido que se geste la igualdad entre la mujer y el hombre.

En contraposición a lo planteado, en el último siglo y lo que va de desarrollo del presente, se han gestado iniciativas que han permitido que la mujer gane espacios en la sociedad, en consecuencia, no puede considerarse como suficiente tal situación, por el 
contrario, deben emplearse mecanismos que afiancen en la sociedad, la igualdad, respeto, de los derechos de la mujer en favor de encontrarse libre de violencia. En Venezuela en los últimos años se ha venido construyendo un marco legal que protege la dignidad de la mujer en todas sus expresiones, siendo así, una oportunidad jurídica para que las féminas puedan acceder a la lucha por defender y hacer velar sus derechos fundamentales como la vida, libertad e igualdad de condiciones sociales y al trabajo.

Esto implica ver a la mujer como un ser humano en toda la dignidad que representa la palabra humanidad y no por el contrario, verla como una esclava sexual o social a merced de mercaderes que por tener concepciones vejadas pretenden subestimar a la mujer. Dar un giro en la concepción que atenta contra la mujer en la sociedad venezolana, genera un trabajo diario de las fuerzas públicas que tienen que ver directamente en defender a la mujer.

En este sentido, se hace necesario respaldar tales acciones con medidas que diversifiquen el accionar que el Estado Venezolano tiene como defensa de la mujer, constituyéndose como alternativa, la creación en las comunidades de los comités jurídicos en defensa de la mujer en derecho a una vida libre de violencia. Tal comité permitirá no solo que la mujer cuando sufra actos de violencia en su contra, cuente con un espacio donde denunciar y apoyarse, sino, que a través del comité se pueda gestionar una educación preventiva como medida alternativa para trabajar en la erradicación de la violencia en contra de la mujer, de lo que se trata es prevenir por medio de la educación que se pueda brindar desde el comité a la par que se atenderán las victimas que pudiesen asistir.

El presente ensayo gestiona la propuesta de crear comités jurídicos como entes comunitarios que puedan brindar el apoyo formativo, legal, psíquico, sanitario, cultural, económico, entre otros, a la mujer como artistas que le permitan articularse hacia el crecimiento integral como persona en beneficio del progreso de la sociedad venezolana. Estos comités tienen en principio mayor relevancia en los sectores sociales más vulnerables, es decir, donde existe mayor pobreza tanto cultural como económica, generando así la posibilidad de contar con espacios de ayuda para la transformación de 
la mujer, esto no como una visión asistencialista del comité en donde la mujer perciba un refugio paternalista, sino, como un medio que le permitirá crecer para ser productiva e independiente en pro de su propio desarrollo, así como el de la sociedad en general.

\section{HACIA UNA VIDA LIBRE DE VIOLENCIA}

La sociedad está constituida por personas e instituciones que hacen vida en la misma, generalmente en esta convivencia se desarrollan relaciones de poder donde las clases dominantes buscan oprimir a las que consideran más débiles, ocasionando así; pobreza, desigualdad, opresión, violencia, entre otros factores negativos para el desarrollo integral del ser humano.

Quizás uno de los casos más típicos en las sociedades ha sido la violencia en contra de la mujer, donde la cultura patriarcal ha interpuesto su dominio con el fin de ocupar la mayor cantidad de espacios de poder. Esto hace que en muchos casos la mujer sea tratada con menosprecio y por debajo del valor que se merece como persona, lo cual, genera compartimientos que derivan en diversos actos de violencia contra la misma.

De ahí, que la mujer progresivamente a lo largo de la historia se ha manifestado para expresar sus derechos, para proponer la igualdad de género como una medida para minimizar o acabar con la violencia de la cual es objeto en diversas ocasiones. Esta lucha del hombre en contra de la mujer por hacerse ver como el fuerte socialmente, trae consigo un sin número de violaciones a los derechos fundamentales de la mujer.

La violencia generada por el hombre en contra de la mujer, ha originado que ésta se despierte y proponga alternativas para promover la defensa de sus derechos; siendo uno de los primeros casos documentados la Declaración de los Derechos Humanos de la Mujer y la Ciudadana en 1791; donde se desarrollaban preceptos, entre los cuales se pueden citar:

Artículo I. La mujer nace libre y permanece igual al hombre en derechos. Las distinciones sociales no pueden estar basadas más que en la utilidad común. Artículo III. El principio de toda soberanía reside, esencialmente, en la Nación, que no es sino la reunión de la mujer y del hombre; ninguna corporación, ningún individuo puede ejercer autoridad alguna que no emane expresamente de ella. Artículo IV. La libertad y la justicia consisten en devolver todo cuanto pertenece a los demás; así pues, el ejercicio de los 
derechos naturales de la mujer no tiene más limitaciones que la tiranía perpetua a que el hombre la somete; estos límites deben ser modificados por las leyes de la naturaleza y de la razón.

En los artículos citados, puede verse como la mujer busca la igualdad con el hombre dentro del rol que ambos deben cumplir en la sociedad, lo cual implica que es necesario que exista una justicia donde se respete a la mujer como ser que brinda y aporta en la construcción de la sociedad. Es de destacar, que esta declaración no fue aprobada y por el contrario su precursora "Olympe de Gouges", fue muerta en la guillotina por quienes expresaban el poder en la Francia de la época.

Por lo tanto; no se logró que se aprobará el respeto a la mujer, así como una serie de derechos que buscaban brindarle dignidad como persona dentro de la sociedad, sino que por el contrario, se vulneró el principal derecho del ser humano como es la vida, solo por el hecho de expresar la necesidad de que fueran reconocidos principios de vida fundamentales en la mujer como parte importante para el progreso de la sociedad. Así mismo; se puede señalar que la Declaración Universal de los Derechos Humanos (1948), expresa en su Artículo 1: "Todos los seres humanos nacemos libres y con los mismos derechos y dignidad. Puesto que tenemos razón y conciencia, debemos tratarnos siempre con respeto". Esto implica que tanto hombre como mujer son iguales en los roles que deben desempeñar en la sociedad, ambos son seres humanos en capacidad de asumir roles protagónicos para el desarrollo de la humanidad.

Es un derecho humano universal respetar a la mujer en igualdad de condiciones, esto con el fin de que pueda generarse una sociedad libre de violencia, de maltratos, que denigran la especie humana, por cuanto no se puede menospreciar como hombre el ser desde donde biológicamente se nace, esto es un acto contra la naturaleza que ha privilegiado a la mujer con el mayor de las bondades que pueda existir como lo es concebir un hijo en su vientre.

En Venezuela, en los últimos años ha surgido un movimiento que busca proteger a la mujer mediante leyes que la igualen con el hombre en los roles que se deben desempeñar como personas y ciudadanos en la sociedad venezolana. En este sentido; la Ley Orgánica Sobre El Derecho de Las Mujeres a Una Vida Libre de Violencia (2007), señala en su: 
Artículo 1. Objeto. La presente Ley tiene por objeto garantizar y promover el derecho de las mujeres a una vida libre de violencia, creando condiciones para prevenir, atender, sancionar y erradicar la violencia contra las mujeres en cualquiera de sus manifestaciones y ámbitos, impulsando cambios en los patrones socioculturales que sostienen la desigualdad de género y las relaciones de poder sobre las mujeres, para favorecer la construcción de una sociedad justa democrática, participativa, paritaria y protagónica.

Los derechos por la defensa e igualdad están consagrados en las leyes venezolanas, de ese modo, existe un marco jurídico que brinda seguridad y apoyo a las mujeres con el fin de que puedan hacer velar sus derechos en momentos en que estos pretendan ser violentados. Se hace necesario que las mujeres venezolanas se organicen para conocer las leyes que se encuentran en su defensa con el fin de apoyarse organizada en función de contar con el apoyo suficiente para fortalecer espacios que le permitan ir ganando en igualdad ante los actos discriminatorios a los cuales pueden ser objeto por parte de una cultura patriarcal - machista que amenaza con invadir en violencia la paz de la mujer venezolana.

Es menester conocer y entender lo que es la violencia en contra de la mujer, con el fin de tener claridad cuando son violentados sus derechos, de ese modo, podrá acudir a instancias que le brinden el apoyo necesario para promover la defensa de su dignidad. Este sentido; la Ley Orgánica Sobre El Derecho de Las Mujeres a Una Vida Libre de Violencia (2007), define a la violencia en su:

Artículo 14. La violencia contra las mujeres a que se refiere la presente Ley, comprende todo acto sexista que tenga o pueda tener como resultado un daño o sufrimiento físico, sexual, psicológico, emocional, laboral, económico o patrimonial; la coacción o la privación arbitraria de la libertad, así como la amenaza de ejecutar tales actos, tanto si se producen en el ámbito público como en el privado.

La violencia en contra de la mujer, representa una serie de acciones que son penadas por la Ley, sea cual sea la forma en la cual se violente la dignidad de la mujer, ésta se encuentra en su derecho de hacer valer su talante como persona en favor de generar una aptitud social de respeto hacia la mujer como coprotagonista del desarrollo social de la nación. 
Sin embargo en Venezuela, hoy día se siguen violentando los derechos de la mujer, se visualizan casos donde se desarrolla una violencia hasta tal punto de causar la muerte. Esto implica que aún falta mucho por educar e informar en el país sobre las políticas que en defensa de la mujer existen. Esta educación no solo debe ser desarrollada en los hombres con el fin de que respeten a la mujer, sino, que debe ser aún más profunda en la población femenina, por cuanto esta es quien en ocasiones, desconoce las leyes y derechos que la amparan para su desarrollo pleno en la sociedad venezolana.

Ante la diversidad de violencia que existen, una mujer puede recibir agresión psicológica, ante lo cual, su autoestima puede verse comprometida e ir disminuyendo cada día más, situación que de un modo u otro es aprovechada por el agresor para controlar de una manera negativa a la mujer, hasta tal punto que pueda ser causa de llevarla hasta la muerte.

Esta situación invita a reflexionar sobre la convivencia que se desarrolla en los hogares, en las relaciones de parejas, más aún, debido al número de acontecimientos anónimos de violencia en contra de la mujer que pueden estar ocurriendo en nuestra sociedad, siendo los mismos silenciados por ignorancia, miedo, falta de apoyo, factores que hacen posible que las mujeres callen y vivan en su soledad el dolor de la violencia doméstica o social, lo cual implica tomar acciones que contribuyan a minimizar la violencia de género en contra de la mujer venezolana. En este sentido; el Observatorio de los derechos humanos de las mujeres (2014) señala que

En el marco de la celebración del Día Internacional de la Eliminación de la Violencia contra la Mujer, el Observatorio Venezolano de los Derechos Humanos de las Mujeres reitera la necesidad de avanzar en la búsqueda de mecanismos que permitan erradicar esta forma de vulneración del derecho de todas y todos a una vida libre de violencia

Uno de estos mecanismos podrían ser las organizaciones gubernamentales y no gubernamentales que se dedican a la defensa de los derechos de la mujer. Sin embargo, se hace necesario que estos se encuentren en todos los sectores, barrios, urbanizaciones, de la geografía venezolana con el fin de que las mujeres puedan tener 
a la mano, un apoyo legal en los momentos que puedan ser vulnerados sus derechos por la violencia de genero.

Prosiguiendo con lo planteado, es necesario constituir en los sectores de la sociedad venezolana comités jurídicos en defensa de la mujer en derecho a una vida libre de violencia, de ese modo, podrán contar con un espacio comunal donde sea apoyadas en momentos de que puedan sufrir violencia, pero sobre todo, educadas y formadas en hacer valer, respetar, sus derechos, ante que estos sean vulnerados, violentados por una sociedad machista que aún no reconoce el valor de la mujer como un ser en igualdad de condición al hombre para contribuir en el progreso integral de la nación.

\section{VIOLENCIA CONTRA LA MUJER}

La violencia de género o contra la mujer, tiene diversas implicaciones sobre la persona quien la sufre, así como su vínculo afectivo inmediato también podría verse comprometido por un accionar negativo que el hombre ejerce sobre la mujer. En tal sentido; Ley orgánica sobre el derecho de las mujeres a una vida libre de violencia (2007) define a la violencia contra la mujer del siguiente modo:

La violencia contra las mujeres a que se refiere la presente Ley, comprende todo acto sexista o conducta inadecuada que tenga o pueda tener como resultado un daño o sufrimiento físico, sexual, psicológico, emocional, laboral, económico o patrimonial; la coacción o la privación arbitraria de la libertad, así como la amenaza de ejecutar tales actos, tanto si se producen en el ámbito público como en el privado.

Esto implica que la violencia en contra de la mujer presenta diversos tipos de agresiones, por lo tanto, el agresor puede desarrollar diversos modos de ataque en contra de la mujer, haciendo que sufra como persona, desvirtuando así la dignidad humana. Es interesante estudiar los tipos de agresión con la finalidad de conocer sus causas, consecuencias, debido que de ese modo, se podrá establecer un proceso educativo y de atención a la víctima por violencia de género o maltrato a la mujer. Por otro lado; La Organización Mundial de la Salud (2014) señala que las Naciones Unidas definen la violencia contra la mujer como 
Todo acto de violencia de género que resulte, o pueda tener como resultado un daño físico, sexual o psicológico para la mujer, inclusive las amenazas de tales actos, la coacción o la privación arbitraria de libertad, tanto si se producen en la vida pública como en la privada.

Esto implica que la mujer puede ser agredida de diversos modos y formas, las cuales no solo se limitan a agresiones físicas o sexuales, sino que existe un conjunto de acciones que perjudican la dignidad de la mujer y violentan sus derechos fundamentales. En tal sentido, la Ley orgánica sobre el derecho de las mujeres a una vida libre de violencia (2007), tipifica los diferentes tipos de violencia, en su Artículo 15:

1.- Violencia psicológica: Es toda conducta activa u omisiva ejercida en deshonra, descrédito o menosprecio al valor o dignidad personal, tratos humillantes y vejatorios, vigilancia constante, aislamiento, marginalización, negligencia, abandono, celotipia, comparaciones destructivas, amenazas y actos que conllevan a las mujeres víctimas de violencia a disminuir su autoestima, a perjudicar o perturbar su sano desarrollo, a la depresión e incluso al suicidio.

2.- Acoso u hostigamiento: Es toda conducta abusiva y especialmente los comportamientos, palabras, actos, gestos, escritos o mensajes electrónicos dirigidos a perseguir, intimidar, chantajear, apremiar, importunar y vigilar a una mujer que pueda atentar contra su estabilidad emocional, dignidad, prestigio, integridad física o psíquica, o que puedan poner en peligro su empleo, promoción, reconocimiento en el lugar de trabajo o fuera de él.

3.- Amenaza: Es el anuncio verbal o con actos de la ejecución de un daño físico, psicológico, sexual, laboral o patrimonial con el fin de intimidar a la mujer, tanto en el contexto doméstico como fuera de él.

4.- Violencia física: Es toda acción u omisión que directa o indirectamente está dirigida a ocasionar un daño o sufrimiento físico a la mujer, tales como: Lesiones internas o externas, heridas, hematomas, quemaduras, empujones o cualquier otro maltrato que afecte su integridad física.

5.- Violencia doméstica: Es toda conducta activa u omisiva, constante o no, de empleo de fuerza física o violencia psicológica, intimidación, persecución o amenaza 
contra la mujer por parte del cónyuge, el concubino, ex cónyuge, ex concubino, persona con quien mantiene o mantuvo relación de afectividad, ascendientes, descendientes, parientes colaterales, consanguíneos y afines.

6.- Violencia sexual: Es toda conducta que amenace o vulnere el derecho de la mujer a decidir voluntaria y libremente su sexualidad, comprendiendo ésta no sólo el acto sexual, sino toda forma de contacto o acceso sexual, genital o no genital, tales como actos lascivos, actos lascivos violentos, acceso carnal violento o la violación propiamente dicha.

7.- Acceso carnal violento: Es una forma de violencia sexual, en la cual el hombre mediante violencias o amenazas, constriñe a la cónyuge, concubina, persona con quien hace vida marital o mantenga unión estable de hecho o no, a un acto carnal por vía vaginal, anal u oral, o introduzca objetos sea cual fuere su clase, por alguna de estas vías.

8.- Prostitución forzada: Se entiende por prostitución forzada la acción de obligar a una mujer a realizar uno o más actos de naturaleza sexual por la fuerza o mediante la amenaza de la fuerza, o mediante coacción como la causada por el temor a la violencia, la intimidación, la opresión psicológica o el abuso del poder, esperando obtener o haber obtenido ventajas o beneficios pecuniarios o de otro tipo, a cambio de los actos de naturaleza sexual de la mujer.

9.- Esclavitud sexual: Se entiende por esclavitud sexual la privación ilegítima de libertad de la mujer, para su venta, compra, préstamo o trueque con la obligación de realizar uno o más actos de naturaleza sexual.

10.- Acoso sexual: Es la solicitud de cualquier acto o comportamiento de contenido sexual, para sí o para un tercero, o el procurar cualquier tipo de acercamiento sexual no deseado que realice un hombre prevaliéndose de una situación de superioridad laboral, docente o análoga, o con ocasión de relaciones derivadas del ejercicio profesional, y con la amenaza expresa o tácita de causarle a la mujer un daño relacionado con las legítimas expectativas que ésta pueda tener en el ámbito de dicha relación.

11.- Violencia laboral: Es la discriminación hacia la mujer en los centros de trabajo: públicos o privados que obstaculicen su acceso al empleo, ascenso o estabilidad en el 
mismo, tales como exigir requisitos sobre el estado civil, la edad, la apariencia física o buena presencia, o la solicitud de resultados de exámenes de laboratorios clínicos, que supeditan la contratación, ascenso o la permanencia de la mujer en el empleo. Constituye también discriminación de género en el ámbito laboral quebrantar el derecho de igual salario por igual trabajo.

12.- Violencia patrimonial y económica: Se considera violencia patrimonial y económica toda conducta activa u omisiva que directa o indirectamente, en los ámbitos público y privado, esté dirigida a ocasionar un daño a los bienes muebles o inmuebles en menoscabo del patrimonio de las mujeres víctimas de violencia o a los bienes comunes, así como la perturbación a la posesión o a la propiedad de sus bienes, sustracción, destrucción, retención o distracción de objetos, documentos personales, bienes y valores, derechos patrimoniales o recursos económicos destinados a satisfacer sus necesidades; limitaciones económicas encaminadas a controlar sus ingresos; o la privación de los medios económicos indispensables para vivir.

13.- Violencia obstétrica: Se entiende por violencia obstétrica la apropiación del cuerpo y procesos reproductivos de las mujeres por personal de salud, que se expresa en un trato deshumanizador, en un abuso de medicalización y patologización de los procesos naturales, trayendo consigo pérdida de autonomía y capacidad de decidir libremente sobre sus cuerpos y sexualidad, impactando negativamente en la calidad de vida de las mujeres.

14.- Esterilización forzada: Se entiende por esterilización forzada, realizar o causar intencionalmente a la mujer, sin brindarle la debida información, sin su consentimiento voluntario e informado y sin que la misma haya tenido justificación, un tratamiento médico o quirúrgico u otro acto que tenga como resultado su esterilización o la privación de su capacidad biológica y reproductiva.

15.- Violencia mediática: Se entiende por violencia mediática la exposición, a través de cualquier medio de difusión, de la mujer, niña o adolescente, que de manera directa o indirecta explote, discrimine, deshonre, humille o que atente contra su dignidad con fines económicos, sociales o de dominación. 
16.- Violencia institucional: Son las acciones $u$ omisiones que realizan las autoridades, funcionarios y funcionarias, profesionales, personal y agentes pertenecientes a cualquier órgano u ente público que contrariamente al debido ejercicio de sus atribuciones, retarden, obstaculicen o impidan que las mujeres tengan acceso a las políticas públicas y ejerzan los derechos previstos en esta Ley, para asegurarles una vida libre de violencia.

17.- Violencia simbólica: Son mensajes, valores, iconos, signos que transmiten y reproducen relaciones de dominación, desigualdad y discriminación en las relaciones sociales que se establecen entre las personas y naturalizan la subordinación de la mujer en la sociedad.

18.- Tráfico de mujeres, niñas y adolescentes: Son todos los actos que implican su reclutamiento o transporte dentro o entre fronteras, empleando engaños, coerción 0 fuerza, con el propósito de obtener un beneficio de tipo financiero u otro de orden material de carácter ilícito.

19.- Trata de mujeres, niñas y adolescentes: Es la captación, el transporte, el traslado, la acogida o la recepción de mujeres, niñas y adolescentes, recurriendo a la amenaza o al uso de la fuerza o de otras formas de coacción, al rapto, al fraude, al engaño, al abuso de poder o de una situación de vulnerabilidad o la concesión o recepción de pagos o beneficios para obtener el consentimiento de una persona que tenga autoridad sobre mujeres, niñas o adolescentes con fines de explotación, tales como prostitución, explotación sexual, trabajos o servicios forzados, la esclavitud o prácticas análogas a la esclavitud, la servidumbre o la extracción de órganos.

En tal sentido, la ley venezolana vigente tiene previsto diecinueve (19) causas para ser sancionadas como violencia a la mujer, esto implica una diversidad de acciones que pueden ser ejercidas por la féminas para hacer valer sus derechos cuando estos son alterados o vilipendiados por su pareja o por terceras personas, según sea el tipo de agresión.

Tal situación crea una complejidad en el accionar de la Ley en beneficio de la mujer, por cuanto no es solo por agresiones físicas o verbales, por las cuales solo se ve afectada, sino, que al existir diversos motivos, estos deben ser conocidos por las mujeres para 
hacer velar sus derechos. Sin embargo, el solo conocimiento de la Ley no implica que puedan acceder a defenderse o hacer respetar sus derechos, implica crear una cultura ciudadana que gestione la defensa de la mujer y la erradicación de la violencia sobre la misma.

Para esto se hace necesario crear mecanismos alternos a la Ley para que esta se pueda cumplir, teniendo un protagonismo importante, los comités en defensa de la mujer, organismos auxiliares de la administración pública, así como ONG dedicados a la defensa de la mujer. La sumatoria de entes que propicien la defensa de la mujer, hacen posible que pueda crearse una cultura que promueva la defensa de los derechos de la mujer, sobre todo de la no violencia a la fémina, esto con la finalidad de coadyuvar en desarrollar una sociedad armónica basada en valores para la paz y la vida como esencia de la existencia humana.

\section{COMITÉ JURÍDICO EN DEFENSA DE LA MUJER EN DERECHO A UNA VIDA LIBRE DE VIOLENCIA}

El comité jurídico es un este que procura la oportunidad de apoyar a la mujer integralmente, especialmente para que se forme para el trabajo productivo como una persona exitosa y pueda hacerse independiente para sostenerse a sí misma y a los hijos que pueda tener, contribuir en el fortalecimiento de su hogar y hacerse respetar de su pareja, por cuanto la unión matrimonial o la convivencia en concubinato, trae consigo una relación reciproca donde ambos (hombre - mujer) tienen los mismos deberes y derechos. Por consiguiente, por medio del comité jurídico es educar a la mujer y a las familias para que puedan establecerse como la institución central de la sociedad venezolana.

Es así, que en el comité jurídico se velará porque los programas sociales destinados a fortalecer la familia venezolana, en especial a la mujer, sean desarrollados para el beneficio del colectivo, de ese modo, las políticas públicas vigentes en la República Bolivariana de Venezuela que garantizan la vida y dignidad humana, podrán cumplirse por medio de estas organizaciones comunales (comités jurídicos en defensa de la mujer), por lo tanto, de lo que se trata es de organizar a la comunidad para que pueda 
hacer velar sus derechos y formarlos para que cumplan con sus deberes, además de apoyarlos en los momentos que necesiten de la ayuda producto de la violencia sufrida por parte de su pareja.

De ese modo, el comité jurídico, estará adscrito al Instituto Nacional de la Mujer, con independencia para actuar en el ámbito comunitario, contando con una coordinación general, encargada de trabajar en conjunto con los demás equipos de trabajo en función de brindar el mejor servicio posible a la colectividad, entre los equipos de trabajo se contará con apoyo legal, apoyo psicológico, desarrollo comunitario, formación al trabajo y a la familia; con lo cual se espera constituir una organización que gestione una sociedad libre de violencia en la mujer.

\section{Visión del comité jurídico}

Constituirse en un órgano auxiliar de las políticas del Estado Venezolano en razón de defender a la mujer de la violencia de género o de cualquier tipo de acción que amenace su integridad como persona, así como ser un ente donde la mujer encuentra un espacio que le permita formarse como ciudadana en razón de brindar lo mejor de sí para el desarrollo integral de la nación.

\section{Misión del comité jurídico}

Trabajar día a día en la formación de una mujer apegada a los principios de igualdad, justicia, paz, que las leyes vigentes fomentan, así como la corriente feminista que iguala a la mujer en deberes y derechos en la sociedad, siendo un órgano para el encuentro en la construcción de una sociedad libre de violencia hacia la mujer

\section{Marco legal}

El comité jurídico en defensa de la mujer, tiene su fundamento legal en la Constitución de la República Bolivariana de Venezuela, Plan de la Patria 2013 - 2019, Ley Orgánica Sobre El Derecho de Las Mujeres a Una Vida Libre de Violencia (2007), Declaración Universal de los Derechos Humanos (1948), Instituto Nacional de la Mujer. 


\section{Coordinación general del comité jurídico}

Se encargará de gestionar ante los entes públicos y privados, los recursos necesarios para el buen funcionamiento del comité, así mismo, debe planificar con los equipos de trabajo para realizar un proceso sistemático que brinde repuestas oportunas a la colectividad que necesita de los servicios del comité.

\section{Apoyo legal}

Este apartado del comité permitirá que las mujeres puedan encontrar orientación y apoyo en relación a los aspectos legales que defienden y promueven sus derechos. Para tal fin, se contará con asesorías legales que gestionen ante los organismos competentes, las denuncias que sean formuladas por violencia en contra de la mujer, lo que permitirá que las féminas que no pueden costearse los servicios jurídicos, tengan la oportunidad de acceder a estos, con lo cual, la violencia podría ser minimizada debido que al saber que cuentan con el apoyo necesario, podrán tener valor de formular las denuncias y proseguir el curso de estas, de acuerdo a los procedimientos vigentes para tal fin.

\section{Apoyo psicológico}

Esta sección del comité se encargará de orientar a las mujeres y a su cuadro familiar en relación a los procesos que deriven de la violencia, así como también se le brindará información para prevenirla. Desde la sección de psicología, se trabajará en crear pensamientos positivos por medio de técnicas y terapias psicológicas que le permitan a la víctima de violencia, elevar su estima, personalidad, para que pueda comprender que es una persona con derecho a ser feliz, teniendo en ella misma la responsabilidad de trabajar para lograrlo. Así mismo; desde esta sección se fomentará la escuela de familia como una alternativa para lograr un núcleo familiar operativo, para que pueda trabajar en ser una organización de paz para la construcción del progreso integral de la nación. 


\section{Desarrollo comunitario}

Una vez que las mujeres sean atendidas por el departamento legal y psicológico del comité, podrán contribuir con el desarrollo comunitario de su comunidad, desde una visión que involucra la superación personal, económica, social. Para esto, se realizarán alianzas con el Instituto Nacional de la Mujer, el cual a su vez, servirá de puente para que las mujeres accedan a los diversos programas de desarrollo socio económico que tiene el gobierno nacional, regional, municipal; de ese modo, las mujeres que han sido víctimas podrán tener un apoyo que les impulse a trabajar para lograr tener una familia solvente desde el punto de vista moral, psicológico, económico, social.

\section{Formación al trabajo y a la familia}

Es una sección del comité dedicada a la formación para el trabajo, así como de la familia, no solo en áreas relacionadas a la violencia, sino, que procura brindar formación integral por medio de la oferta de diplomados que gestionen la capacitación permanente como medio para crecer como persona y como grupo social. De lo que se trata con este equipo de trabajo, es la de prevención de la violencia, es decir, trabajar con aquellas mujeres y familias que no han sufrido violencia, entre las cuales pueden estar adolescentes, jóvenes, estudiantes, público en general, que tengan la de formarse para crecer integralmente.

\section{Referencias Consultadas}

1. Bayona, J. (2014). Violencia contra la mujer. Marco histórico evolutivo y predicción del riesgo. Tesis doctoral. Universidad Autónoma de Madrid. España.

2. Campo, M. (2004). Intersubjetiva en la orientación de la violencia familiar. Tesis de Grado. La Universidad del Zulia. Facultad de Humanidades y Educación. División de Estudios para Graduados. Programa de Doctorado en Ciencias Humanas. Maracaibo, Venezuela.

3. Declaración Universal de los Derechos Humanos (1948). Derecho a la vida. Documento en línea. Disponible en: http://www.un.org/es/universal-declarationhuman-rights/. Consultado el 13-5-17. 
4. Declaración de los Derechos Humanos de la Mujer y la Ciudadana en 1791. La Declaración de los Derechos de la Mujer y la Ciudadana. Documento en línea. Disponible en: https://igualamos.wordpress.com/2012/10/26/declaracion-de-losderechos-de-la-mujer-y-la-ciudadana-olympia-de-gouges/ Consultado el 13-5-17.

5. Labrador, J. (2010). Consecuencias psicológicas de la violencia de género. Documento en línea. Disponible en: http://medicablogs.diariomedico.com/reflepsiones/2010/07/21/consecuenciaspsicologicas-de-la-violencia-de-genero/. Consultado el: 13-5-17.

6. López, L. (2011). Violencia hacia la mujer por su pareja. Intervenciones orientadas a su manejo integral. Universidad Ciencias Médicas Cienfuegos. Tesis doctoral. Cienfuegos, Cuba.

7. Observatorio de los derechos humanos de las mujeres (2014). Violencia de género. Documento en línea. Disponible en: http: http://observatorioddhhmujeres.org/. Consultado el 13-5-17.

8. Organización maltrato enfermería (2011). Violencia de género. Documento en línea. Disponible en: http://maltratoenfermeria.blogspot.com/2011/10/tipos-deviolencia-de-genero.html. Consultado el 13-5-17.

9. Organización Mundial de la Salud (2014). Violencia contra la mujer. Documento en línea. Disponible en: http://www.who.int/mediacentre/factsheets/es/. Consultado el 13-5-17.

10. Ramírez, A. (2012). Causas que generan la violencia en contra la mujer de acuerdo a la ley orgánica sobre el derecho a las mujeres a una vida libre de violencia. Tesis de grado. Facultad de ciencias jurídicas y políticas. Escuela de derecho. Universidad José Antonio Páez. San Diego, Venezuela.

11.Sotjany, C. (2006). Rasgos de personalidad en mujeres sometidas a violencia doméstica y mujeres que no lo son. Tesis (Psicólogo). Universidad Rafael Urdaneta. Facultad de Ciencias Políticas, Administrativas y Sociales. Maracaibo, Venezuela.

12.La Organización Mundial de la Salud (2014). Violencia contra las mujeres. Documento en línea.

Disponible en: 
http://www.un.org/es/events/endviolenceday/pdfs/unite the situation sp.pdf. Consultado el 13-5-17.

\section{Ley Orgánica Sobre El Derecho de Las Mujeres a Una Vida Libre de Violencia} (2007). Definición y formas de violencia contra las mujeres. Documento en línea. Disponible en: http://www.derechos.org.ve/pw/wp-content/uploads/11.-LeyOrg\%c3\%a1 nica-sobre-el-Derecho-de-las-Mujeres-a-una-Vida-Libre-deViolencia.pdf. Consultado el 13-5-17.

(C2018 por los autores. Este artículo es de acceso abierto y distribuido según los términos y condiciones de la licencia Creative Commons Attribution (http://creativecommons.org/licenses/by/4.0/). 